\title{
北红尾鸲鸣唱句子内结构分化及利用其鸣唱识别个体
}

\author{
黄亚灵, 杨 青, 蒋 纯, 夏灿玮* \\ （北京师范大学 生命科学学院, 北京 100875)
}

摘要：鸣唱具有吸引异性和驱赶同性的功能，受到性别间选择和性别内选择的共同影响。在少数鸟类中, 不 同的句子或同一句子的不同部分分别侧重执行吸引异性和驱赶同性的功能, 句子的结构也相应的出现了分化。该 研究于 2011 年繁殖季对北京小龙门地区 32 只北红尾鸲(Phoenicurus auroreus)的鸣唱分析发现, 其鸣唱的句子可以 分为差异显著的两个部分: 稳定部分和可变部分。这两个部分的语图相关变量在均值和变异系数上均有显著差异 (配对样本 $t$-检验, $P<0.05$ )。其中稳定部分在个体内非常保守, 而可变部分个体内存在较大的变化。在指出句子结 构分化的基础上, 利用北红尾鸲鸣唱的稳定部分进行个体识别, 获得了较高的正确率。

关键词: 北红尾鸲; 鸣唱; 稳定部分; 可变部分; 个体识别

中图分类号：Q959.739;S864;Q62 文献标志码：A 文章编号：0254-5853-(2012)03-0249-06

\section{Two distinct parts within the song of Phoenicurus auroreus, and individual identification on the basis of the song}

\author{
HUANG Ya-Ling, YANG Qing, JIANG Chun, XIA Can-Wei* \\ (College of Life Science, Beijing Normal University, Beijing 100875, China)
}

\begin{abstract}
Bird song has two key functions: attraction and competition. The structures of these songs have been shaped by both intra- and intersexual selection. Accordingly, there are some species that appear to use different parts of song to attract females and repel males. Alongside this functional separation, the structure of song can also have significant divergence. We studied the songs from 32 individuals of Phoenicurus auroreus, recorded in Beijing Xiaolongmen forest Park during the 2011 breeding season. The song of Phoenicurus auroreus consists of two distinct parts, a stereotyped and variable part. Significant differences between these two parts were observed between all the variables we measured. Based on the stereotyped part, the rate of correct individual identification is satisfactory, and most of songs can be correctly classified.
\end{abstract}

Key words: Phoenicurus auroreus; Song; Stereotyped part; Variable part; Individual identification

声信号是动物通讯交流的重要手段，尤其在鸟 类的生活史中起着重要的作用(Gill, 2007)。鸟类的 发声可分为鸣叫(call)和鸣唱(song)两类。鸣叫通常 简短而单调, 几乎所有鸟类都可以发出, 多用在特 定的场合，如乞食、报警; 鸣唱通常委婉而复杂，多 数为处于繁殖期的雀形目 (鸣禽)雄鸟发出, 用以保 卫领域、吸引配偶(Catchpole \& Slater, 2008; Wang et al, 2003)。一般认为, 鸟类鸣唱的主要功能有: 同性 竞争和异性吸引。巫瑀(Emberiza)、树莺属(Cettia)、 苇莺属(Acrocephalus)、林莺属(Dendroica) 等的部分
鸟类，可分别利用不同的鸣唱句子执行这两个功能 (Brunner \& Pasinelli, 2010), 并且伴随着句子功能的 分化，句子的结构也出现明显的差异，如日本树莺 (Cettia diphone)用于自发鸣唱吸引异性的句子在哨 音部分(whistle portion)音节数较少、音量较大，而 用于驱赶同性的句子在哨音部分音节数较多、音量 较小 (Park \& Park, 2000); 芦州 (Emberiza schoeniclus) 吸引异性的句子与驱赶同性的句子在 持续时间、复杂程度上有着显著差别, 吸引异性的 句子结构相对简单, 但持续时间更长(Brunner \&

收稿日期: 2011-12-26; 接受日期: 2012-04-06

基金项目: 国家自然科学基金基础科学人才培养基金能力提高项目(野外实践) (J0830630/J0106)

*通信作者(Corresponding author), E-mail: xiacanwei@126.com 
Pasinelli, 2010)。

野外观测和回放实验证实，多数情况下，雄鸟 鸣唱的每个句子都可以同时起到同性竞争和异性 吸引的作用(Kroodsma \& Byers，1991; Marier \& Slabbekoorn, 2004); 但在同一个句子的不同部分是 否存在功能上的分化, 即句子的不同部分分别编码 同性竞争和异性吸引的信息, 目前实证还较少, 仅 见于荣科(Sylviidae)少数鸟类(Collins et al, 2009; Fessl \& Hoi, 1996)的报道。同一句子的不同部分分 别执行同性竞争和异性吸引的功能, 受到性间选择 (intrasexual selection) 和 性 内选择 (intersexual selection)的压力不同, 导致功能分化的同时也出现 结构的分化(Collins et al, 2009), 如黑顶林苜(Sylvia atricapilla)句子前半部分侧重执行吸引异性的功能, 其频宽较大、结构复杂; 句子的后半部分主要执行 驱赶同性的功能, 其频宽较窄、结构简单(Collins et al，2009)。又如须苇茑(Acrocephalus melanopogon) 句子起始部分传达同性竞争的信息，结构相对简单， 而句子结尾部分侧重吸引异性, 其结构相对复杂 (Fessl \& Hoi, 1996)。

鸟类个体间由于遗传、发育和所处环境的不同, 导致了发声器官结构的细微变化或发声习惯的差 异(Gilbert et al, 1994), 从而导致鸣声在传递信息的 同时也表达出个体的特征(Fox, 2008)。野外研究发 现, 鸟类可以通过鸣唱对同种的不同个体进行识别, 如亲戚识别(Sharp et al, 2005)和邻居识别(Wei et al, 2011)。在研究中通过鸟类鸣声来识别和监测个体, 作为一种非损伤、低干扰性的标记方法，也有着很 广泛的应用前景(Terry et al, 2005), 如濒危物种保 护(Delport et al, 2002)、生活史研究(Laiolo et al, 2007)、种群数量监测(Hartwig, 2005)等。

北红尾鸲(Phoenicurus auroreus)隶属于雀形目 (Passeriformes) 鸫科 (Turdidae) 红尾鸲属 (Phoenicurus), 广布于欧亚大陆东部, 是我国华北 地区常见的夏候鸟。该鸟喜筑巢于屋檐下的墙缝、 农电土坎的凹陷处, 在繁殖季雄鸟常站在屋檐、电 线、树梢等视野开阔处发出响亮的鸣唱(Cai，1987; $\mathrm{Wu}, 1986)$ 。为此, 本文首先通过研究北红尾鸲的鸣 唱特征，着重突出该鸟鸣唱的语句内可分为结构差 异显著的稳定部分 (stereotyped part)和可变部分 (variable part), 为进一步研究北红尾鸲鸣唱语句内 功能的分化提供基础资料; 其次, 在指出句子内结 构分化的基础上, 尝试利用句子稳定部分进行个体 识别(individual identification), 为更好地监测、研究 该物种，提供方法借鉴。

\section{1 材料和方法}

\section{1 研究地区}

野外工作在小龙门林场及其周边进行, 研究地 位于北京西部门头沟区 $\left(\mathrm{N} 40^{\circ} 00^{\prime}, \mathrm{E} 115^{\circ} 26^{\prime}\right)$, 属太行 山脉小五台山余脉。夏季高温多雨, 冬季寒冷干燥, 为温带半湿润季风性气候。年平均降水量 500 700 $\mathrm{mm}$, 年平均气温 $2 \sim 7^{\circ} \mathrm{C}$ 。主要生境类型有农田、灌 从、落叶阔叶林、人工针叶林和亚高山灌从草甸等 (Qin et al, 2011; Xia et al, 2011b)。北京师范大学在 该地区开展山地动物野外实习已近 30 年(19832011 年), 对其动植物资源有长期的积累资料。北红 尾鸲为该地区夏候鸟，巢筑于石隙、墙壁或林区房 子内，亦见于树洞或人工巢箱内筑巢。由于北红尾 鸲在居民点附近分布集中，所以挑选 4 个居民点(林 场场部、小龙门村、齐家庄、洪水口村)进行野外声 音采集工作。这 4 个居民点之间相距约 2 $\sim \mathrm{km}$ 。

\section{2 野外工作}

野外工作于 2011 年北红尾鸲的繁殖季进行, 其中 6 月 18-26 日调查研究点北红尾鸲的数量和 分布, 6 月 27-29 日进行录音。采用 TASCAM HD-P2 便携式数字录音机 (TASCAM, 日本), 和 Sennheiser MKH416 P48 外接强指向性话筒 (SENNHEISER ELECTRONIC, 德国)录音, 采样精 度(sample width)设置为 16 Bit, 采样频率(sampling frequency)设置为 $44.1 \mathrm{kHz}$ (Xia et al, 2010)。为获得 高质量的录音, 录音时距目标个体的距离 $<10 \mathrm{~m}$ 。为 避免假重复, 每个录音个体之间的距离相隔足够远 (如位于不同的居民点), 或是能同时观测到多个鸣 唱的个体, 以确保不会重复录音。共获得了 32 只个 体清晰可供分析的录音。其中林场场部 6 只，小龙 门村 11 只, 齐家庄 10 只, 洪水口村 5 只。

\section{3 声音量化}

利用 Goldwave5.25 声音处理软件(GoldWave Inc, 加拿大)过滤 $1 \mathrm{kHz}$ 以下背景噪音, 并以 22.05 $\mathrm{kHz}$ 重新采样。利用 Avisoft-SASLab Pro 5.1.09 software 声音分析软件(Avisoft Bioacoustics, 德国) 生成语图, 参数设置为: 快速傅里叶变换(FFT length $)=256$ points，哈明窗口 (Hamming window), 帧长(frame size) $=100 \%$, 重叠 (overlap) $=50 \%$, 频率分 辨率 (frequency resolution) $=172 \mathrm{~Hz}$, 时间分辨率 
(time resolution) $=2.9 \mathrm{~S}$ 。参照 $\mathrm{Qu}$ et al, (2011) 和 Xiao et al, (2008)的研究, 本文中使用的鸣声术语如下。

音素(note): 语图上表现为一段连续的曲线, 是 鸣唱的最基本单位。

句子(song): 包含多个音素的连续段落，句子间 通常有明显的间隔。

仿照 Lindholm \& Linden(2007)的声音量化方法, 我们对每个北红尾鸲挑选 10 句连续的录音进行测 量。测量的变量包括句子的持续时间 $\left(t_{\text {Dur }}\right)$ 、句子的 音素数 $\left(N_{\mathrm{b}}\right)$ 、句子的最高频率 $\left(F_{\max }\right)$ 、句子的最低频 率 $\left(F_{\min }\right)$ 、句子起始的频率 $\left(F_{\text {sta }}\right)$ 、句子结尾的频率 $\left(F_{\text {end }}\right)$ 。在发现北红尾鸲鸣唱的句子可分为稳定部分 (stereotyped part)和可变部分(variable part)(详细介 绍见本文结果部分)后, 为了比较这两个部分结构 的差异, 我们分别测量了这两部分的持续时间 $\left(t_{\text {Dur_s }}, t_{\text {Dur_v }}\right)$ 、最高频率 $\left(F_{\text {max_s }}, F_{\text {max_v }}\right) 、$ 最低频率 $\left(F_{\text {min s }}, F_{\text {min v }}\right)$ 、音素数 $\left(N_{\mathrm{bs} s}, N_{\mathrm{bvv}}\right)$ 以及两部分之间的 间隔时间 $\left(t_{\mathrm{Int}}\right)$ 。我们还分别计算了整个句子以及稳 定部分和可变部分的频宽 ( $\left.F_{\text {band, }}, F_{\text {band_s, }}, F_{\text {band_v }}\right)$ (最 高频率与最低频率的差值)。共 18 个变量(图 1)。

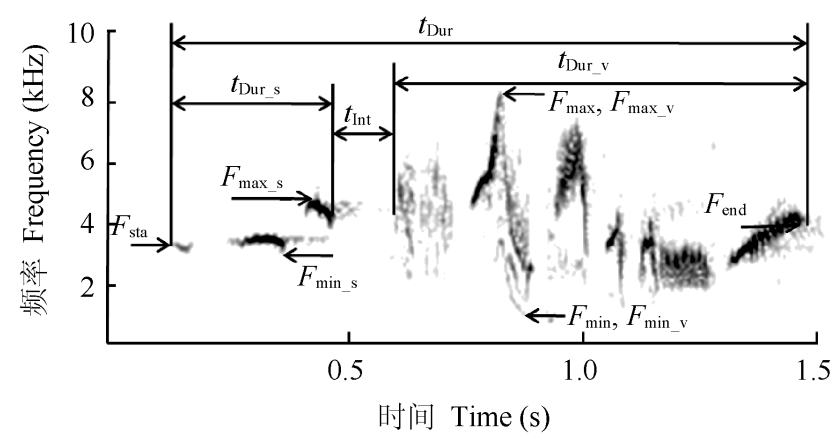

图 1 测量变量的示意图

Fig. 1 Method of measuring the variables

\section{4 数据分析}

计算变量在个体内的均值和变异系数。在北红 尾鸲鸣唱特征的描述性统计时, 利用每个个体的均 值和变异系数计算总体的均值和平均变异系数。在 确定所有变量均符合正态分布后(单样本 $K-S$ 检验, $P>0.05$ ), 利用配对样本 $t$-检验, 比较稳定部分和可 变部分相关变量的差异。

为了进一步探究稳定部分和可变部分在个体 内和个体间变异的差异, 仿照 Xia et al(2011a)的研 究, 我们使用了语图比较分析 (spectrographic cross-correlation)。语图比较分析通过比较两段语图
的结构计算出这两段声音的相似性系数，该系数介 于 0 至 1 之间, 数值越高相似性越大。相比于通过 测量变量量化声音的分析方法，语图比较分析可以 更全面的利用声音的信息。首先，32 个个体的每个 个体截取 10 句清晰的语图, 并将其分为稳定部分 和可变部分，共计获得 640 段语图 $(10 \times 2 \times 32)$; 然 后分别对稳定部分和可变部分进行相似性计算, 语 图间两两比较, 共获得 102080 个相似性系数 $(2 \times 320(320-1) / 2)$ 。最后利用双样本 $K-S$ 检验比较 个体内语图相似性系数和个体间语图相似性系数 的分布差异。按照我们的观察句子内的稳定部分在 个体内不同语句间变异很小，我们预测在分布上稳 定部分的相似性系数在个体内和个体间会有明显 差别，且个体内的相似性系数数值更大。

利用语句稳定部分的语图比较分析结果进行 鸣声的个体识别研究。首先计算句子识别到个体的 正确率。采用刀切法(Jack-knifed)的过程，每个句子 依次选取一次作为待识别的句子。计算待识别的句 子与某一个体发出的所有句子(不包括待识别句子 本身)相似性系数的均值，做为该待识别句子与该 个体的相似性系数。将该待识别句子归类到相似性 系数最高的个体。比较该待识别句子实际发出的个 体与上述方法归类的个体是否一致，以检验句子识 别到个体的正确性(Xia et al, 2011a)。输出结果为所 有句子正确识别到个体的比率(Terry et al, 2005; Xia, 2010)。在计算句子识别正确率的基础上，我们进一 步计算可以利用鸣唱正确识别的个体的比率。如果 一个个体正确识别到该个体的句子数超过错误归 类到任一其他个体的句子数, 则定义该个体可以正 确识别。输出结果为可以正确识别个体的比率。

语图比较分析利用 Avisoft-SASLab Pro 4.36 software 自带的 Corr32 模块执行; 统计分析利用 R2.11.1 (R Development Core Team)完成。数据用 “mean $\pm S D$ ”的形式表示。所有检验在 $P<0.05$ 时认 为有显著差异。

\section{2 结 果}

\section{1 北红尾鸲繁殖期鸣唱特征}

北红尾鸲鸣唱句子的最高频率 $(6.66 \pm 0.44) \mathrm{kHz}$, 最低频率 $(2.12 \pm 0.28) \mathrm{kHz}$ ，频宽 $(4.54 \pm 0.61) \mathrm{kHz}$ ，句 子起始的频率 $(3.72 \pm 0.32) \mathrm{kHz}$, 句子结尾的频率 $(3.51 \pm 0.50) \mathrm{kHz}$ ，句子持续时间 $(1.61 \pm 0.22) \mathrm{s}$ ，由 (10.61 \pm 1.48$)$ 个音素构成(表 1)。 
表 1 北红尾鸲的鸣唱特征

Tab. 1 Song characteristics of Phoenicurus auroreus

\begin{tabular}{cccccc}
\hline $\begin{array}{c}\text { 变量名 } \\
\text { Variables }_{(\mathrm{kHz})}\end{array}$ & $\begin{array}{c}\text { 均值 } \\
\text { Mean }\end{array}$ & $\begin{array}{c}\text { 标准差 } \\
\text { Standard } \\
\text { deviation }\end{array}$ & $\begin{array}{c}\text { 最大值 } \\
\text { Maximum }\end{array}$ & $\begin{array}{c}\text { 最小值 } \\
\text { Minimum }\end{array}$ & $\begin{array}{c}\text { 样本量 } \\
\text { Sample } \\
\text { size }\end{array}$ \\
\hline$F_{\max }$ & 6.60 & 0.44 & 7.56 & 5.74 & 32 \\
$F_{\min }$ & 2.12 & 0.28 & 2.85 & 1.50 & 32 \\
$F_{\text {band }}$ & 4.54 & 0.61 & 5.73 & 3.57 & 32 \\
$F_{\text {sta }}$ & 3.72 & 0.32 & 4.15 & 2.74 & 32 \\
$F_{\text {end }}$ & 3.51 & 0.50 & 4.66 & 2.57 & 32 \\
$t_{\text {Dur (s) }}$ & 1.61 & 0.22 & 2.08 & 1.18 & 32 \\
$N_{\mathrm{b}}$ & 10.61 & 1.48 & 13.70 & 8.00 & 32 \\
\hline
\end{tabular}

\section{2 北红尾鸲鸣唱稳定部分和可变部分的差异}

北红尾鸲鸣唱的句子可以分为结构差异明显 的两个部分: 稳定部分(stereotyped part)和可变部分 (variable part) (图 2)。其中稳定部分位于句子的前半 段, 持续时间 $(0.35 \pm 0.08) \mathrm{s}$, 由(3.38 \pm 0.72$)$ 个音素构
成; 固定部分位于句子的后半段，持续时间 $(0.98 \pm 0.20) \mathrm{s}$, 由(7.21 \pm 1.34$)$ 个音素构成; 两者之间 有 $(0.29 \pm 0.06) \mathrm{s}$ 的时间间隔。稳定部分和可变部分 不仅在变量的均值上差异显著(配对样本 $t$-检验, $P<0.05)$, 而且在变量的变异系数上也有明显差异 (配对样本 $\mathrm{T}$ 检验, $P<0.05$ )(表 2)。如表 2 所示，可变 部分在个体内的变异系数要显著大于稳定部分在 个体内的变异系数。

语图比较分析结果可见，稳定部分在个体内的 相似性系数 $(0.64 \pm 0.16)$ 与个体间的相似性系数 $(0.35 \pm 0.15)$ 在分布上差异显著(双样本 $K-S$ 检验, $P<0.05)$ (图 3A), 两者均值之差为 0.29 。可变部分相 似性系数在个体内 $(0.22 \pm 0.08)$ 和个体间 $(0.19 \pm 0.06)$ 的分布虽然差异也很显著 (双样本 $K-S$ 检验, $P<0.05$ ) (图 3B), 但两者均值之差仅为 0.03 。
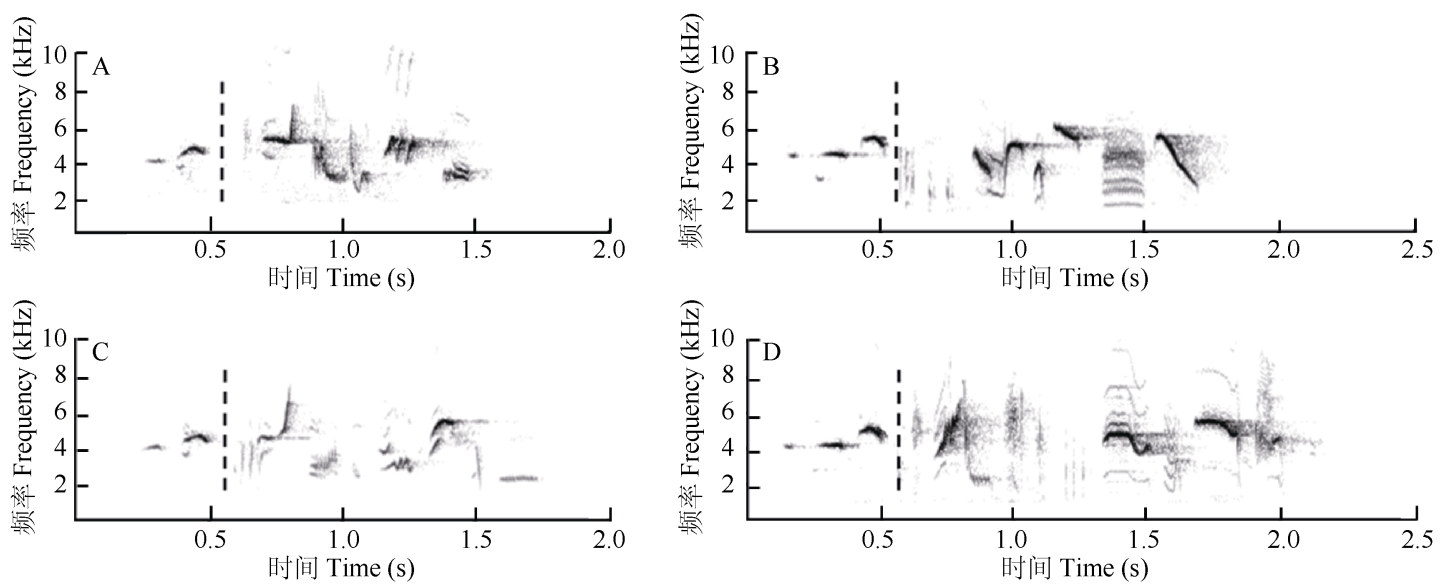

图 2 北红尾鸲鸣唱的语图

Fig. 2 Spectrograms of Phoenicurus auroreus

$\mathrm{A} 、 \mathrm{C}$ 由同一个体发出; B、D 由另一个体发出; 虚线之前的为句子的稳定部分; 虚线之后的为句子的可变部分。

A, C from one individual; B, D from another individual; the spectrograms before dashed line represent stereotyped part, while the spectrograms after dashed line represent variable part

表 2 北红尾鸲鸣唱稳定部分和可变部分鸣声变量的比较

Tab. 2 Characteristics of stereotyped part and variable part of Phoenicurus auroreus

\begin{tabular}{|c|c|c|c|c|c|}
\hline $\begin{array}{c}\text { 变量名 Variables } \\
(\mathrm{kHz})\end{array}$ & $\begin{array}{c}\text { 均值沶准差 } \\
\text { Mean } \pm \text { Standard deviation }\end{array}$ & $\begin{array}{c}\text { 配对样本 } t \text {-检验 Paired } \\
\text { samples } t \text {-test } \\
\end{array}$ & $\begin{array}{c}\text { 变异系数的均值 } \\
\text { Mean of variation coefficient }\end{array}$ & $\begin{array}{c}\text { 配对样本 } t \text {-检验 } \\
\text { Paired samples } t \text {-test }\end{array}$ & $\begin{array}{c}\text { 样本量 } \\
\text { Sample size }\end{array}$ \\
\hline$F_{\text {max } \_}$ & $5.21 \pm 0.22$ & $P<0.05$ & 0.03 & $P<0.05$ & \multirow{2}{*}{32} \\
\hline$F_{\text {max_v }}$ & $6.58 \pm 0.48$ & $(t=-14.53)$ & 0.14 & $(t=-7.50)$ & \\
\hline$F_{\text {min_s }}$ & $3.46 \pm 0.54$ & $P<0.05$ & 0.05 & $P<0.05$ & \multirow{2}{*}{32} \\
\hline$F_{\text {min_v }}$ & $2.10 \pm 0.29$ & $(t=13.98)$ & 0.19 & $(t=-7.59)$ & \\
\hline$F_{\text {band_s }}$ & $1.76 \pm 0.57$ & $P<0.05$ & 0.12 & $P<0.05$ & \multirow{2}{*}{32} \\
\hline$F_{\text {band_v }}$ & $4.48 \pm 0.63$ & $(t=-19.82)$ & 0.22 & $(t=-4.87)$ & \\
\hline$t_{\text {Dur_s }}(\mathrm{s})$ & $0.35 \pm 0.08$ & $P<0.05$ & 0.11 & $P<0.05$ & \multirow{2}{*}{32} \\
\hline$t_{\text {Dur_v }}(\mathrm{s})$ & $0.98 \pm 0.20$ & $(t=-17.42)$ & 0.35 & $(t=-8.80)$ & \\
\hline$N_{\text {b_s }}$ & $3.38 \pm 0.72$ & $P<0.05$ & 0.08 & $P<0.05$ & \multirow{2}{*}{32} \\
\hline$N_{\mathrm{b}_{\mathrm{v}} \mathrm{v}}$ & $7.21 \pm 1.34$ & $(t=-13.70)$ & 0.34 & $(t=-8.84)$ & \\
\hline
\end{tabular}



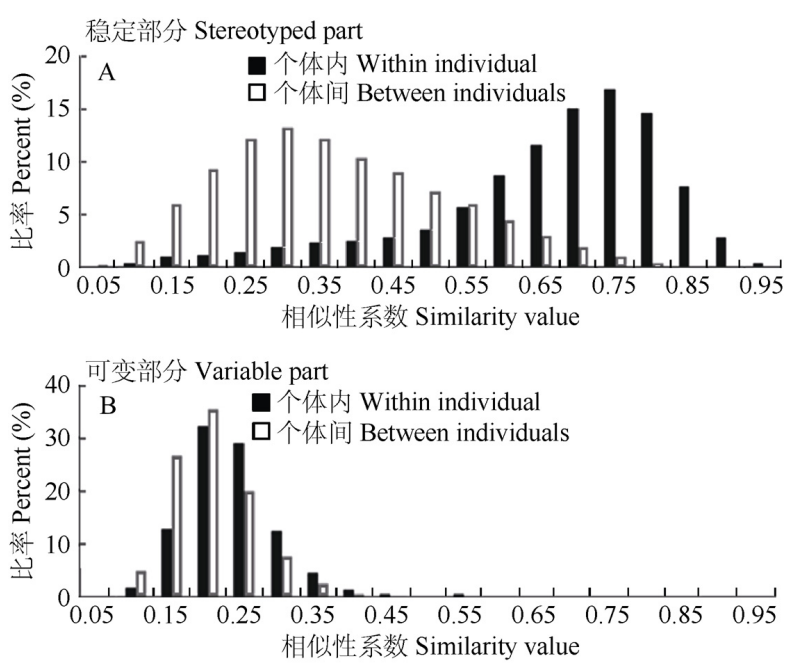

图 3 语图比较分析得出的鸣声相似性系数

Fig. 3 The similarity value calculated by spectrographic cross-correlation

\section{3 利用鸣声个体识别}

利用句子稳定部分进行个体识别，有 234 句正 确识别到发出的个体，占句子总数(320)的 $73.1 \%$; 有 29 个个体正确识别到该个体的句子数超过错误 归类到任一其他个体的句子数, 即可以利用鸣唱正 确识别该个体，占个体总数(32)的 $90.6 \%$ 。

\section{3 讨 论}

\section{1 北红尾鸲繁殖期鸣唱句子内的结构分化}

本研究发现, 北红尾鸲鸣唱的语句内存在结构 差异显著的两个部分。稳定部分频宽窄、音素数少、 持续时间短，在个体内的不同语句间极少有变化、 十分保守，而可变部分频宽宽、音素数多、持续时 间长, 在个体内的不同语句间, 无论从时间变量还 是频率变量上看, 都有着极显著的变化(图 2、表 2)。 鸟类鸣唱具有吸引异性和驱赶同性的作用, 其结构 必将受到来自同性选择和来自异性选择压力的共 同影响(Berglund et al, 1996; Catchpole \& Slater, 2008; Marier \& Slabbekoorn, 2004)。同性选择和异性 选择对鸣唱结构的塑造方式在不同的鸟类中存在 较大的差异(Byers, 2011; Byers \& Kroodsma, 2009), 但通常认为复杂的鸣唱有利于吸引异性(Soma \& Zsolt Garamszegi, 2011), 而简单的鸣唱有利于种内 识别, 在驱赶同性中起到重要作用(Collins et al, 2009; Irwin, 2000)。须苇莺和黑顶林莺鸣唱的语句 内也存在结构差异明显的两个部分, 其中结构简单 的部分主要用来驱赶同性, 而结构相对复杂的部分 主要用来吸引异性(Collins et al, 2009; Fessl \& Hoi,
1996)。参照须苇莺和黑顶林莺的例子，我们推测北 红尾鸲鸣唱相对简单的稳定部分主要用于同性竞 争, 而相对复杂的可变部分主要用于异性吸引。并 提出以下预测：1) 北红尾鸲配对前(鸣唱更多的用 于吸引异性)相比于配对后(鸣唱更多的用于保卫领 域), 鸣唱的可变部分将更加复杂, 而稳定部分基本 保持不变; 2) 删除句子内可变部分(屏蔽了异性吸 引的功能), 在回放实验中仍可诱发雄鸟保卫领域 的反应，而不易吸引雌鸟靠近，反之亦然。

\section{2 利用北红尾鸲鸣唱的稳定部分进行个体识别}

本研究利用北红尾鸲鸣唱的稳定部分进行个 体识别, $73.1 \%$ 的句子可以被正确识别到发出的个 体，90.6\%的个体正确识别到该个体的句子数超过 错误归类到其他个体的句子数：即利用鸣声可以较 好地识别北红尾鸲个体。鸟类的鸣声往往同时要传 达多重的信息，如反映种间识别的信息 (species information)、反映出生地的信息(local dialect)、反 映个体质量的信息(quality information), 以及反映 个体的信息(individual information)。利用鸣声进行个 体识别已在过百种的鸟类中得到验证，但个体特征 是否可能相对集中的编码于鸣唱的某一部分, 目前 关注的还较少(Fox, 2008; Terry et al, 2005)。Wegrzyn et al (2009)发现大苇莺(Acrocephalus arundinaceus) 鸣唱语句的起始部分在个体内较为保守, 相对集中 的体现了个体特征。本研究发现, 北红尾鸲鸣唱的 稳定部分在个体内十分保守, 并可以较好的进行个 体识别, 丰富了个体特征可集中编码于鸣唱某一部 分的实证。

在实际研究中应用鸣声个体识别, 还需要满足 两个条件：1)鸣声个体性的特征需要保持时间上的 稳定性；2)随着个体数的增多, 鸣声识别的正确性 仍然要足够高(Terry et al, 2005)。对于鸣声特征在时 间上的稳定性，在乌林鸮(Strix nebulosa) (Rognan et $\mathrm{al}$, 2009)、蚁斩(Formicarius moniliger) (Kirschel et al, 2011)等鸟类上已得到验证, 即用于短期区分个体 的鸣声特征, 也可以很好的在较长的时间段上(繁 殖季的不同时期，或是不同的繁殖季)区分个体。北 红尾鸲鸣声个体特征是否也可以保持时间上的稳 定性, 还有待对于标记个体的长期监测。对于条件 2)个体数量增多对鸣声个体识别正确性的影响, 对 北红尾鸲的作用很有限。由于北红尾鸲喜欢在居民 点附近占区、繁殖, 其个体数量必然受到居民点大 小的限制, 在同一个居民点不易出现大量个体, 从 
而影响鸣声个体识别正确率。由于居民点之间往往 间隔数公里，而且鸟类在繁殖季有占有并保卫领域 的习性，所以不同居民点间的北红尾鸲依据地理位 置即可以做出初步的区分。

\section{参考文献:}

Berglund A, Bisazza A, Pilastro A. 1996. Armaments and ornaments: An evolutionary explanation of traits of dual utility [J]. Biol J Linn Soc, 58(4): 385-399.

Brunner P, Pasinelli G. 2010. Variation in singing style use in the reed bunting Emberiza schoeniclus: Influencing factors and possible functions [J]. J Avian Biol, 41(4): 388-397.

Byers BE. 2011. Birdsong, migration and sexual selection: a skeptical view [J]. Anim Behav, 82(5): E1-E3.

Byers BE, Kroodsma DE. 2009. Female mate choice and songbird song repertoires [J]. Anim Behav, 77(1): 13-22.

Cai QK. 1987. Birds of Beijing [M]. Beijing: Beijing Publishing. [蔡其㑆. 1987. 北京鸟类志 $[\mathrm{M}]$. 北京: 北京出版社.]

Catchpole CK, Slater PJB. 2008. Bird Song: Biological Themes and Variations. [M]. 2nd ed. New York: Cambridge University Press.

Collins SA, de Kort SR, Pérez-Tris J, Tellería JL. 2009. Migration strategy and divergent sexual selection on bird song [J]. Proc R Socy B: Biol Sci, 276(1656): 585-590.

Delport W, Kemp AC, Ferguson JWH. 2002. Vocal identification of individual African wood owls Strix woodfordii: A technique to monitor long-term adult turnover and residency [J]. Ibis, 144(1): 30-39.

Fessl B, Hoi H. 1996. The significance of a two part song in the moustached warbler (Acrocephalus melanopogon) [J]. Ethol Ecol Evol, 8(3): 265-278.

Fox EJS. 2008. A new perspective on acoustic individual recognition in animals with limited call sharing or changing repertoires [J]. Anim Behav, 75(3): 1187-1194.

Gilbert G, McGregor PK, Tyler G. 1994. Vocal individuality as a census tool: practical considerations illustrated by a study of 2 rare species $[\mathrm{J}] . J$ Field Ornithol, 65(3): 335-348.

Gill FB. 2007. Ornithology. [M].3rd ed. New York: W. H. Freeman and Company.

Hartwig S. 2005. Individual acoustic identification as a non-invasive conservation tool: an approach to the conservation of the African wild dog Lycaon pictus (Temminck, 1820) [J]. Bioacoustics, 15(1): 35-50.

Irwin DE. 2000. Song variation in an avian ring species [J]. Evolution, 54(3): 998-1010.

Kirschel ANG, Cody ML, Harlow ZT, Promponas VJ, Vallejo EE, Taylor CE. 2011. Territorial dynamics of Mexican ant-thrushes Formicarius moniliger revealed by individual recognition of their songs [J]. Ibis, 153(2): 255-268.

Kroodsma DE. Byers BE. 1991. The function(s) of bird song [J]. Integr Comp Biol, 31(2): 318-328.

Laiolo P, Vöegeli M, Serrano D, Tella JL. 2007. Testing acoustic versus physical marking: two complementary methods for individual-based monitoring of elusive species [J]. J Avian Biol, 38(6): 672-681.

Lindholm A, Lindén A. 2007. Some notes on the distribution and songs of two Oriental Cuckoo taxa, Cuculus (saturatus) saturatus and Cuculus (saturatus) optatus [J]. Forktail, 23(1): 12-16.

Marier PR. Slabbekoorn H. 2004. Nature's Music: The Science of Birdsong [M]. Oxford: Elsevier Academic Press.
致谢: 北京师范大学生命科学院张雁云教授在 实验设计、数据分析和写作方面给予指导; 匿名审 稿人对文章的修改提出了宝贵意见，在此一并表示 诚挚地谢意!

Park SR. Park D. 2000. Song type for intrasexual interaction in the bush warbler [J]. Auk, 117(1): 228-232.

Qin X, Wang YX, He T, Wei CT, Xia CW. 2011. Song characteristics of chestnut-lined rock bunting (Emberiza godlewskii)[J]. J Beijing Normal Univ (Nat Sci Ed), 47(4): 405-408.[覃歆, 王乙霞, 何田, 魏 晨蹈, 夏灿玮. 2011. 灰眉岩雨 (Emberiza godlewskii) 的鸣唱特征 分析. 北京师范大学学报: 自然科学版, 47(4): 405-408.]

Qu WH, Li F, Sha JB, Zhang YM. 2011. Analyzing Japanese marsh warbler (Megalurus pryeri) song behavior in the breeding season [J]. Zool Res, 32(2): 141-149. [曲文慧, 李枫, 沙剑斌, 张玉铭. 2011. 斑背大尾莺 繁殖期鸣声行为分析. 动物学研究, 32(2): 141-149]

Rognan CB, Szewczak JM, Morrison ML. 2009. Vocal Individuality of great gray owls in the Sierra Nevada [J]. J Wildlife Manage, 73(5): 755-760.

Sharp SP, McGowan A, Wood MJ, Hatchwell BJ. 2005. Learned kin recognition cues in a social bird [J]. Nature, 434(7037): 1127-1130.

Soma M, Zsolt Garamszegi LZ. 2011. Rethinking birdsong evolution: meta-analysis of the relationship between song complexity and reproductive success [J]. Behav Ecol, 22(2): 363-371.

Terry AMR, Peake TM, McGregor PK. 2005. The role of vocal individuality in conservation[J]. Front Zool, 2(1): 10

Wegrzyn E, Leniowski K, Osiejuk TS. 2009. Introduce yourself at the beginning-possible identification function of the initial part of the song in the Great Reed Warbler Acrocephalus arundinaceus [J]. Ornis Fennica, 86(2): 61-70.

Wang AZ, Lei FM, Jia ZY. 2003. Female choice and evolution of male songs in birds[J]. Zool Res, 24(4): 305-310. [王爱真, 雷富民, 贾志云. 2003. 雌性选择与雄鸟鸣唱的进化. 动物学研究, 24(4): 305-310.]

Wei M, Lloyd H, Zhang YY. 2011. Neighbour-stranger discrimination by yellow-bellied tit Parus venustulus: evidence for the "dear-enemy" effect [J]. J Ornithol, 152(2): 431-438.

Wu ZK. 1986. The Avifauna of Guizhou [M]. Guiyang: Guizhou People's Publishing House. [吴至康. 1986. 贵州鸟类志. 贵阳: 贵州人民出版社.]

Xia CW. 2010. Song Structure and Variation in the Song of Cettia fortipes [D]. Thesis, Beijing Normal University, Beijing. [夏灿玮. 2010. 强脚 树莺 (Cettia fortipes) 鸣唱的研究. 硕士学位论文, 北京师范大学, 北京.]

Xia CW, Huang R, Wei CC, Nie PW, Zhang YY. 2011a. Individual identification on the basis of the songs of the Asian Stubtail (Urosphena squameiceps) [J]. Chn Birds, 2(3): 132-139.

Xia CW, Wang YX, Qin X, He T, Zhang YY. 2011b. Spatial pattern of song sharing within Emberiza godlewskii population [J]. Chn J Ecol, 30(6): 1131-1136.[夏灿玮，王乙霞，覃歆，何田，张雁云. 2011b. 灰眉岩幽 种群内鸣声的空间格局. 生态学杂志, 30(6): 1131-1136.]

Xia CW, Xiao H, Zhang YY. 2010. Individual variation in brownish-flanked bush warbler songs[J]. Condor, 112(3): 591-595.

Xiao H, Zhou ZX, Wang N, Zhang YY. 2008. Analyzing song characteristics of yellow-bellied tits (Parus venustulus) [J]. Zool Res, 29(3): 277-284. [肖华, 周智金金, 王宁, 张雁云. 2008. 黄腹山雀的鸣唱特征 分析. 动物学研究, 29(3): 277-284.] 